\title{
The ROI of DAM at National Geographic Channels International
}

\section{Eric Marks}

is an independent consultant and author. Previously, he was a client advisor with Cambridge Technology Partners, and before that he was managing director with BrightRoad, a division of Ontos Inc. While at PricewaterhouseCoppers, Marks focused on supply chain management. In his book 'Business Darwinism: Evolve or Dissolve', Marks looks at the make-or-break impact of IT on accelerating the global struggle for market share. His second book, 'Executive's Guide To Web Services', published in April 2004 by Wiley US, untangle the maze of standards, concepts, and terminology that blur Web services and SOA, arming the reader with enabling concepts and providing a solid foundation and reference text with which to unleash untapped business potential.

Keywords: hosted service, subscription DAM, ClearStory, deployment options

Abstract Digital Asset Management (DAM) is rapidly gaining recognition as a key component of an enterprise content management strategy. It is important to recognize the growing need to create, store, retrieve and manage rich media files. DAM solution providers identified this need and are offering a variety of options to manage digital assets, including installed software managed in-house, hosted solutions managed outside the organization or the best of both worlds - systems that begin as hosted solutions and then, as an organization's IT infrastructure becomes more sophisticated, can be easily converted to an installed solution. This paper explores the DAM strategies National Geographic Channels International is deploying and how their business case for DAM considers business model, enterprise adoption rate and the question of inhouse vs outsourced. Hard cost savings, business process efficiency improvements and the ability to take advantage of rapidly emerging market opportunities are contributing factors to ROI and DAM solution justification.

Journal of Digital Asset Management (2006) 2, 287-291. doi:10.1057/palgrave.dam.3650048

\section{INTRODUCTION}

Digital Asset Management (DAM) is rapidly gaining recognition as a key component of an enterprise content management strategy. The proliferation of digital assets - in multiple formats - is presenting significant challenges to IT departments as organizations are coming to realize that systems to manage these assets are essential, valuable and a best business practice.

Recognizing this growing need to create, store, retrieve and manage rich media files, DAM solution providers are offering a variety of options to manage digital assets, including installed software managed in-house, hosted solutions managed outside the organization or the best of both worlds - systems that begin as hosted solutions and then, as an organization's IT infrastructure becomes more sophisticated, can be easily converted to an installed solution.

According to Frost and Sullivan, hosted alternatives are rapidly gaining momentum as the solution of choice over installed systems, as users look to best manage their financial and human capital. For some companies, hosted DAM services are an end in themselves - a deployment alternative that avoids a major investment in infrastructure or the addition of IT staff necessary to manage an enterprise DAM system. Hosting alternatives also allow companies to gain critical capabilities quickly, while they are building out their infrastructures or developing a proof of concept to help refine requirements and demonstrate ROI.

\section{SEEKING THE RIGHT SOLUTION}

In searching for a DAM solution, users are faced with three basic choices - installed software/ in-house management solution hosted or a combination of the two.

Organizations that have a sophisticated IT operation are most likely to choose an in-house solution. They have the hardware, software, 
budget and network infrastructure to support an internal system.

A hosted solution offers significant advantages to companies with constrained IT resources staff and budget - or companies whose usecase requires maximum uptime with the failover and redundancy that a hosted solution can provide.

For some organizations, a compelling alternative is a hosted solution that can seamlessly transition in-house. This allows the organization to implement a pay-as-you-go pilot, launching the solution immediately - and realizing the benefits - while concurrently building out their infrastructure, staffing and modifying requirements to prove maximum value before making the long-term commitment to a large investment.

There are several companies providing hosted solutions including Corbis, through its acquisition of eMotion, but this might not be an option for companies looking to ultimately transition the service to an in-house installed application. Other DAM providers like interwoven, Northplains and Artesia/OpenText offer the alternative of a hosted service through third-party partners. ClearStory Systems touts an enterprise-level system that can be delivered as either a hosted service or an in-house application, and the company manages the hosted service itself, not via a third party.

When choosing an enterprise DAM product and vendor, a system that is available on both a hosted and installed basis provides a best of both worlds scenario in terms of flexibility. But this benefit can only be gained if the system is fully browser-based, with full user functionality and consistent user experience - whether it is hosted or installed. Another point to consider is that a system that can seamlessly transition from a hosted service to an in-house solution accommodates changing business models without interruption.

"Hosted services are rapidly gaining in popularity among a variety of end users primarily because of their versatility and ability to be rapidly deployed", said Mukul Krishna, DAM expert and analyst with Frost and Sullivan.

"Hosted systems are managed by experts, which alleviates the necessity for a massive in-house investment in both staff and equipment. It also gives the organization the ability to better focus their resources on core business efforts."

\section{WORLDWIDE TELEVISION NET- WORK CHOOSES HOSTED SERV- ICES}

National Geographic Channels International (NGCI) provides award-winning cable television programming to more than 230 million subscribers throughout the world. NGCI chose a hosted solution to simplify the management of thousands of hours of program tapes, photos and marketing materials that need to be accessible to its 29 regional offices around the globe.

The DAM system serves as a virtual creative services network for NGCI offices worldwide. NGCI uploads promotional and programming content including still photos, videotapes and scripts into a secure central repository. This central resource enables any office to be the creative hub and the distribution point for marketing materials on behalf of their particular region - and in some cases, to the global group. Access and usage rights are fully controlled by NGCI headquarters. Content within the system is managed by many internal contributors, including creative and marketing staff, for example, who manage collateral content and ensure correct versioning; or the operations team who manage video pasting and whether content is available for regional download.

According to Abigail Grossman, manager of Operations and Technical Services for NGCI, a hosted DAM system was the only answer. Already responsible for a massive broadcast management system, Grossman knew that her organization did not have the resources to manage a complex DAM system.

\footnotetext{
"We really are a 365-day 24/7 operations with worldwide usage, so, we need to rely on our vendor to ensure smooth, efficient, secure operation. The solution we chose was quickly implemented and gives us $24 / 7$ technical support just about anywhere in the world any time of the day or night, something we could never have tackled in-house", said Grossman.

"It also seamlessly manages all content, keeps that content updated, and provides accessibility and secure storage."
} 
Hosting allowed Grossman's organization to get up and running much more quickly than if they had tried to create the same structure in-house. Grossman chose a hosted solution that was not managed by a third party but managed directly by the provider.

"We didn't have to worry about building out the infrastructure and didn't have to worry about acquiring in-house experts to manage the process. We were up and running is just a few months."

A hosted solution is clean and efficient, according to Grossman. There is no software or hardware to buy, no internal maintenance costs, no upgrade or support costs. Patches and upgrades are handled by the hosting vendor. The system can support multiple users in widespread geographic areas and it is highly scaleable.

"Experts configured the system to map to our business model, and they provide support the solution, which has alleviated that pressure from our organization." persistent performance and rapid ROI. More importantly, NGCI needed round-the-clock availability for its global constituency. They required a DAM service provider they could trust to provide high-availability and that would assume the burden of maintaining and upgrading the system as well as provide the management and operational expertise. These functions require real labor investments, which NGCI preferred not to make themselves. For this reason, they selected a vendor who was dedicated to sustaining a state-of-the-art hosting facility and which has a demonstrated commitment to delivering hosted services long-term.

Yet, even if a company ultimately wants to have the solution in-house, opting to start with a hosted service still has numerous advantages. For one, it can help companies build the business case for resource allocation by demonstrating the effectiveness and ROI potential of the solution. This prevents the delays that can occur while struggling to achieve buyin, which can result in significant losses and missed opportunities.

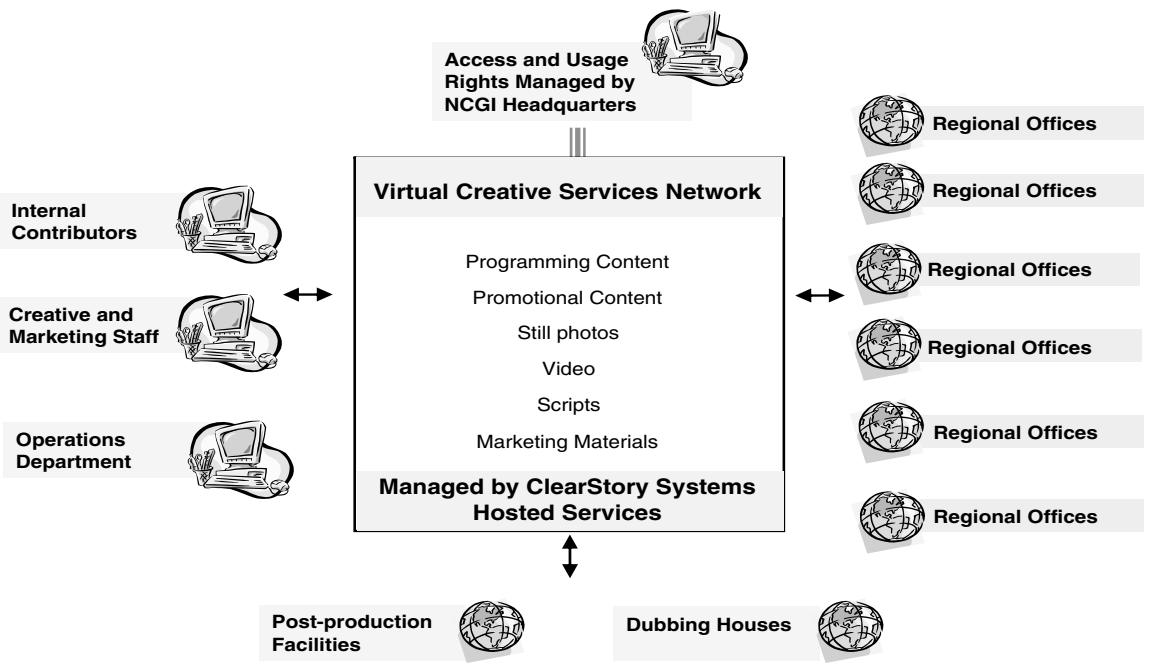

\section{FUNCTIONALITY WITHOUT DIFFICULTY}

When it comes to hosted services, its critical for the solution to pass every test of functionality, security, scalability and performance. Like most companies that consider a hosted service, National Geographic sought quick deployment,
Once implemented, the customer should have complete control of the system through a nontechnical, web-based interface. The system should easily accommodate the addition of new users, metadata definitions, other rich media assets, expanded storage and other administrative changes. And internal system administrators 
should be able to make these changes independent of the hosted service provider.

\section{SECURE, FLEXIBLE, SCALABLE AND AVAILABLE}

Hosted services should be available as a standalone, or able to be integrated with existing enterprise applications, either inside or outside network firewalls. Hosting facilities should feature high-level security including 24/7 monitoring, CCTV surveillance systems, and even biometric entry scanning to ensure maximum protection for valuable customer data. Considering that rich media files are often very large in size, it is important to confirm that the vendor has technology to support high bandwidth for maximum performance, including redundant lines and multiple points of entry.

At NGCI multiple point connectivity is critical for the worldwide group. Their system provides high speed, redundant internet connections.

"If there's an issue with one service provider, service can be seamlessly picked up by another," Grossman said.

Vendors should be able to provide uptime guarantees and offer an infrastructure with redundancy at every key level to ensure automatic recovery in the event of a component or software failure. The provider should also offer a level of redundancy that provides additional protection in the event of a regional disaster or other catastrophic event. Although this level of redundancy, availability, and security would exceed those of a typical in-house implementation, they can be a big benefit of a hosted service.

\section{LOOK FOR VARIABLE OPTIONS}

Look for a vendor that can provide a variety of pricing models, including a pay-as-you-go contract or a licensed software option with managed services. This allows a company to begin with a small pilot project to refine requirements and processes before launching the deployment on a large scale.

Look for a vendor that has extensive expertise in providing both hosted and non-hosted applications. For hosted services, ask the vendor about their hosting facility, its security measures and infrastructure and confirm their commitment to the model.

Look for a vendor that can customize the solution. Their system should be able to handle a full spectrum of media-from video to Photoshop, Quark and other graphic file types.

Look for a vendor that can provide 24/7 service wherever you need it-worldwide.

Insist on scalability, which is important not only for storage and user licenses, but also for the ability of the system to adjust to the growing needs of the organization.

Look for ease of use. You want a solution that embodies all the technical attributes but that which can be easily and efficiently managed by all end users.

Often, a DAM implementation creates new revenue opportunities. For example, a company may discover its library of photography can be repackaged and offered for sale; or an advertising agency may offer DAM as a service to its clients; or a marketing services firm may offer a hosted brand management solution. When revenue generation is a strategic opportunity for a DAM implementation, it is important for the system to provide economy of scale, with the ability to support a multi-tenant hosting model. In this case, there is one underlying infrastructure supporting separate 'companies' or user groups. Imagine the cost advantages of managing multiple clients with one infrastructure stack.

\section{ROI and TCO for on-premise and hosted solutions}

When measuring ROI, a critical consideration is total cost of ownership (TCO). For an onpremise solution, the TCO includes the costs of hardware, software, support, professional services and ongoing administration of the system. If separate business units can utilize the same DAM solution for different applications, TCO is reduced by leveraging the knowledge and/or infrastructure gained through the first deployment. Each additional DAM implementation, therefore, should account for a progressively lower TCO. For an outsourced or hosted DAM solution, while there are no direct equipment costs, you must factor in the ongoing monthly or annual fees. 
A significant benefit of a hosted model is faster deployment, which may allow you to take advantage of a market opportunity sooner, enable an accelerated product launch and realize increased revenue as a result. For example, if an internal deployment is delayed 12-18 months due to infrastructure or other concerns, and your ROI model proves possible returns within 6 months, it makes sense to outsource and begin earning those returns sooner, even if the upfront costs are slightly higher. For example, if your DAM solution costs $\$ 300,000$, with a 6-month projected ROI, the additional $\$ 300,000-\$ 600,000$ in savings justifies deployment sooner rather than later. If a hosted solution is a preliminary phase to a long-term plan for an installed in-house system, be certain to select a solution that can later be installed onsite.

\section{CONCLUSION}

Whether or not hosting is the best financial solution for a company, is based on a company's overall business model, enterprise adoption rate, and resource considerations. Hard cost savings, business process efficiency improvements, and the ability to take advantage of rapidly emerging market opportunities are contributing factors to ROI and DAM solution justification. In the case of NCCI, they have not considered bringing the DAM solution in-house. The benefits of a hosted solution outweigh an internal system, according to Grossman. Specific financial ROI results are proprietary but Grossman says that the system has had a tremendous impact on budget and human resources.

"If you think about the way information was shared prior to the DAM implementation, i.e. shared network drives, overwhelming amounts of email and shipping costs-all that has been eliminated" said Groseman.

"Now there's a central repository where everyone knows they can find the latest version of what they need. It's allowed us to redeploy those people who spent hours just assembling and shipping product and answering emails. The process works so smoothly that we've just about forgotten how bad it was." 